九州大学学術情報リポジトリ

Kyushu University Institutional Repository

Improvement of double-buffer problem in LESRANS interface region by introducing an anisotropy-resolving subgrid-scale model

ABE, Ken-ichi

Department of Aeronautics and Astronautics, Faculty of Engineering, Kyushu University : Professor

http://hdl. hand le. net/2324/4150684

出版情報: Theoretical and Computational Fluid Dynamics. 32 (3)，pp. 263-283，2018-02-07. Springer nature

バージョン :

権利関係 : 


\title{
Improvement of double-buffer problem in LES-RANS interface region by introducing an anisotropy-resolving subgrid-scale model
}

\author{
Ken-ichi ABE
}

Received: date / Accepted: date

\begin{abstract}
The "double-buffer problem" has been regarded as a crucial concern for the strategy behind the hybrid large eddy simulation (LES)/Reynoldsaveraged Navier-Stokes (RANS) model (or HLR model, for short). Such models are likely to show unphysical mean-velocity distributions in the LES-RANS interface region, where "super-streak structures" also appear that look like low-speed streaks generated in the near-wall region of wall turbulence. To overcome this difficulty, the stochastic backscatter model, in which the vortex structures in the interface region are divided into smaller scales, holds promise due to the effect of random source term imposed in the momentum equation. Although this method is effective, several parameters must be prescribed and their specification process is arbitrary and ambiguous. An alternative advanced HLR model has been proposed, in which an anisotropy-resolving subgrid scale (SGS) model was adopted in the LES region as well as a one-equation non-linear eddy-viscosity model in the RANS region. Previous investigations indicated that this HLR model did not exhibit or, at least, largely reduced the "double-buffer problem" in the mean-velocity distribution, with no special treatment being applied. The main purpose of the present study is to reveal why this HLR model improves the predictive performance in the LES-RANS interface region. Specifically, we focus on the role of the extra anisotropic term introduced in the SGS model, finding that it plays an important role in enhancing vortex structures in the interface region, leading to a considerable improvement in model performance.
\end{abstract}

Keywords Turbulence · Large Eddy Simulation · Anisotropy-Resolving SGS Model · Hybrid LES/RANS Model · Interface Region

Ken-ichi ABE

Professor, Department of Aeronautics and Astronautics, Kyushu University, 744 Motooka, Nishi-ku, Fukuoka 819-0395, Japan

Tel.: +81-92-8023023

Fax: +81-92-8023001

E-mail: abe@aero.kyushu-u.ac.jp 


\section{Introduction}

The large eddy simulation (LES) is known to be a useful way to predict complex turbulence in engineering applications. Although a significant number of research groups have proposed several kinds of the subgrid-scale (SGS) models (see for example, [1]-[6]), there still remains a serious difficulty in its application to very high Reynolds-number $(R e)$ flows. For instance, reasonable LES for a channel flow needs a grid resolution such as $\Delta x^{+} \sim 100$ ( $x$ : the streamwise direction) and $\Delta z^{+} \sim 20$ ( $z$ : the spanwise direction) in the near-wall region, as well as $\Delta y^{+} \sim 1$ in the wall-normal direction, where the no-slip condition is specified at the wall surface: here the superscript plus sign ()$^{+}$ signifies a value normalized by the friction velocity $u_{\tau}$. When LES is applied to the channel flow at $R e_{\tau}=10^{4}$, where $\operatorname{Re}_{\tau}\left(=u_{\tau} \delta / \nu\right)$ is the Reynolds number based on the half channel height $(\delta)$, the kinematic viscosity $(\nu)$ and the friction velocity, the grid resolution $\Delta z^{+}=20$ translates into $\Delta z=2 \times 10^{-3} \delta$ (i.e., 500 grid points per $\delta$ in $z$-direction). Such a grid-resolution requirement for high-Re LESs is significant, even if it is applied to simple wall-shear flows.

To solve this problem, one promising approach is the so-called "hybrid LES/RANS (HLR) model," where RANS is the abbreviation for Reynoldsaveraged Navier-Stokes modeling. This model was originally based on the concept of a hybrid model connecting LES with RANS in the near-wall region. To date, many researchers have addressed this challenging problem (see for example, [7]-[16]), a representative model of this type of approach being the "detached eddy simulation (DES)" (Nikitin et al.[8]). DES was studied very actively and an advanced version of DES, called the "delayed detached eddy simulation (DDES)," was proposed to improve model performance (see for example, Spalart et al.[14]). Although most previous HLR models adopted a linear eddy-viscosity form to represent the SGS and Reynolds stresses, Abe[17] proposed an HLR model that introduced a non-linear eddy-viscosity model (NLEVM) to reproduce the near-wall stress anisotropy more accurately.

Although each study provided encouraging results and useful insights into developing HLR models, there still remain several important issues to be solved. Among them, the "double-buffer problem" proved to be a crucial concern. In fact, many HLR models are likely to show unphysical mean-velocity distributions in the LES-RANS interface region, which mainly arise through a lack of modeling the Reynolds-shear (Re-shear) stress, resulting in steeper mean-velocity gradients in the interface region. It is also known that in this region, we often see "super-streak structures" that look like low-speed streaks generated in the near-wall region of wall-turbulence. To overcome this difficulty, Piomelli et al.[18] proposed a stochastic backscatter model that held promise. Because of the effect of the random source term imposed in the momentum equation, the model divides the vortex structures in the interface region into smaller scales. Although this method is effective, several parameters must be determined and their specification process is arbitrary and ambiguous.

As an alternative, Abe[19] recently proposed an advanced HLR model, in which an anisotropy-resolving SGS model[20] was introduced in the LES re- 
gion as well as a non-linear one equation RANS model in the RANS region. Surprisingly, the results as well as those of a previous study[21] indicated that this type of HLR model did not show or, at least, largely reduced the "doublebuffer problem" in the mean-velocity distribution, although no special treatment was applied in the LES-RANS interface region. An important feature of this SGS model is the introduction of an extra anisotropic term (EAT) to represent the SGS stress anisotropy more correctly; although the EAT is modeled not to give any energy transfer between the grid-scale (GS) and SGS parts to maintain computation stable[20][22]. In this sense, this SGS model includes no backscatter effect in the GS-SGS energy transfer, differentiating it from a stochastic backscatter model. With regard to this issue, Ohtsuka and Abe[23] and Abe and Ohtsuka[24] investigated the role of this type of SGS model in enhancing turbulent vortex structures by analyzing using the streamwise vorticity transport equation. They found EAT to be very effective in activating the streamwise vortex structures, even if no backscatter effect is included in the SGS eddy viscosity. In this sense, an EAT in the SGS model is expected to function effectively instead of a stochastic backscatter model introduced in the interface region.

Based on the background provided above, the main purpose of the present study is to investigate the reason why this anisotropy-resolving HLR model[19] improves the predictive performance in the LES-RANS interface region. To illustrate, this HLR model is applied to fully-developed plane channel flows with various grid resolutions. We compare the computational results with and without the EAT to confirm the effectiveness of the EAT for improving the predictive performance. We then analyze the influence of the EAT in the distribution of the $R e$-shear stress that dominates the mean-velocity prediction. Also, the turbulent structures in the LES-RANS interface region developed with different grid resolutions are compared. In particular, we focus on the role of the EAT in determining the primary size of the turbulent structures in the interface region.

\section{Turbulence Model}

2.1 Governing equations and hybrid LES/RANS approach

The filtered (or Reynolds-averaged) governing equations for an incompressible turbulent flow may be written as

$$
\begin{gathered}
\frac{\partial \bar{U}_{i}}{\partial x_{i}}=0 \\
\frac{\mathrm{D} \bar{U}_{i}}{\mathrm{D} t}=-\frac{1}{\rho} \frac{\partial \bar{P}}{\partial x_{i}}+\frac{\partial}{\partial x_{j}}\left\{\nu\left(\frac{\partial \bar{U}_{i}}{\partial x_{j}}+\frac{\partial \bar{U}_{j}}{\partial x_{i}}\right)-\tau_{i j}\right\},
\end{gathered}
$$

where $\overline{(~})$ denotes a filtered value in the LES region or a Reynolds-averaged value in the RANS region, respectively. In Eqs. (1) and (2), $\rho, \bar{P}$ and $\bar{U}_{i}$ denote 
the density, filtered static pressure and filtered velocity, respectively. The SGS stress $\tau_{i j}$ in the LES region is originally expressed as

$$
\tau_{i j}=\overline{U_{i} U_{j}}-\bar{U}_{i} \bar{U}_{j} .
$$

Note that $\tau_{i j}$ coincides with the following general expression for the Reynoldsstress tensor in the RANS region:

$$
\tau_{i j}=\overline{u_{i} u_{j}},
$$

where $u_{i}$ is defined as $u_{i}=U_{i}-\bar{U}_{i}$.

Originally, the HLR model is a turbulence model that uses LES in the region far from the wall; the modeling is then connected with RANS in the near-wall region. A strategy to connect these LES and RANS regions smoothly is the following hybrid approach (see for example, Abe[17]):

$$
\phi=\left(1-f_{h b}\right) \phi_{(R A N S)}+f_{h b} \phi_{(L E S)},
$$

where $\phi$ is a flow variable and $f_{h b}$ is a switching function. In Eq. (5), $f_{h b}$ is close to 1 in the region far from the wall, where LES is adopted. Conversely, as $f_{h b}$ approaches 0 , the RANS calculation is adopted. For instance, if we consider the SGS (Reynolds) stress, the expression used is

$$
\tau_{i j}=\left(1-f_{h b}\right) \tau_{i j(R A N S)}+f_{h b} \tau_{i j}(L E S) .
$$

In the next section, we describe the anisotropy-resolving SGS model (Abe[20][22]) that is adopted for $\tau_{i j(L E S)}$ in Eq. (6). The one-equation non-linear eddyviscosity model (NLEVM) for $\tau_{i j(R A N S)}$ and the switching function $f_{h b}$ used in this study are briefly explained in Appendix.

2.2 An anisotropy-resolving SGS model in the LES region

The SGS stress $\tau_{i j}$ in Eq. (3) is modeled using

$$
\tau_{i j}=\frac{2}{3} k_{S G S} \delta_{i j}-2 \nu_{S G S} S_{i j}+2 k_{S G S} b_{i j}^{E A T},
$$

where $k_{S G S}$ and $\nu_{S G S}$ are the SGS turbulence energy and the SGS eddy viscosity, respectively. The strain-rate tensor $S_{i j}$ is expressed as

$$
S_{i j}=\frac{1}{2}\left(\frac{\partial \bar{U}_{i}}{\partial x_{j}}+\frac{\partial \bar{U}_{j}}{\partial x_{i}}\right) .
$$

In Eq. (7), the anisotropy tensor $b_{i j}^{E A T}$ on the right-hand side is modeled as

$$
b_{i j}^{E A T}=\frac{\tau_{i j}^{\prime}-\left(-2 \nu^{\prime} S_{i j}\right)}{\tau_{k k}^{\prime}-\left(-2 \nu^{\prime} S_{k k}\right)}-\frac{1}{3} \delta_{i j}=\frac{R_{i j}^{\prime}}{\tau_{k k}^{\prime}}, \quad R_{i j}^{\prime}=\tau_{i j}^{\prime}{ }^{a}-\left(-2 \nu^{\prime} S_{i j}\right),
$$

where $\tau_{i j}^{\prime}{ }^{a}=\tau_{i j}^{\prime}-\tau_{k k}^{\prime} \delta_{i j} / 3$. 
In Eq. (9), $\tau_{i j}^{\prime}$ is given by the following representative scale-similarity model of Bardina et al.[25]:

$$
\tau_{i j}^{\prime}=\left(\bar{U}_{i}-\widehat{\bar{U}}_{i}\right)\left(\bar{U}_{j}-\widehat{\bar{U}}_{j}\right)
$$

where ( ) denotes a test-filtered value. In the model, $\nu^{\prime}$ is an equivalent eddy viscosity evaluated using an isotropic EVM-type linear approximation for Eq. (10),

$$
\tau_{i j}^{\prime a} S_{i j}=-2 \nu^{\prime} S_{i j} S_{i j}=-2 \nu^{\prime} S^{2} \quad \longrightarrow \quad \nu^{\prime}=-\frac{\tau_{i j}^{\prime}{ }^{a} S_{i j}}{2 S^{2}}
$$

where $S^{2}=S_{i j} S_{i j}$. As $R_{i j}^{\prime}$ is evaluated by subtracting an isotropic EVM form from the original Bardina et al.[25] model, the extra term in Eq. (7) including $R_{i j}^{\prime}$ is then expected to successfully predict the SGS-stress anisotropy.

Concerning the linear EVM in Eq. (7), we basically adopts the one-equation SGS model proposed by Inagaki[26] with some minor modifications. The SGS viscosity $\nu_{S G S}$ is modeled using

$$
\begin{aligned}
& \nu_{S G S}=C_{S G S} f_{S G S} \sqrt{k_{S G S}} \Delta, \quad f_{S G S}=1-\exp \left\{-\left(\frac{y_{\varepsilon}^{\prime}}{A_{0}}\right)^{4 / 3}\right\}, \\
& y_{\varepsilon}^{\prime}=\left\{\frac{\left(\nu \varepsilon_{S G S}\right)^{1 / 4} y}{\nu}\right\} \sqrt{C_{l} \frac{y}{\Delta}}, \quad C_{S G S}=0.05, \quad A_{0}=30, \quad C_{l}=4,
\end{aligned}
$$

where $\varepsilon_{S G S}$ is the dissipation rate for $k_{S G S}$ and $\Delta$ is the filter width. In this study, $k_{S G S}$ is evaluated using

$$
\begin{gathered}
\frac{\mathrm{D} k_{S G S}}{\mathrm{D} t}=\frac{\partial}{\partial x_{j}}\left\{\left(\nu+\frac{\nu_{S G S}}{\sigma_{S G S}}\right) \frac{\partial k_{S G S}}{\partial x_{j}}\right\}-\tau_{i j} \frac{\partial \bar{U}_{i}}{\partial x_{j}}-\varepsilon_{S G S}, \\
\varepsilon_{S G S}=C_{\varepsilon} \frac{k_{S G S}{ }^{3 / 2}}{\Delta}+\frac{2 \nu k_{S G S}}{y^{2}} .
\end{gathered}
$$

The model constants are specified as $\sigma_{S G S}=0.5$ and $C_{\varepsilon}=0.835$. A more detailed description of the present anisotropy-resolving SGS model is given in $[20]$ and $[22]$.

2.3 Decomposition of modeled stresses into isotropic and anisotropic parts

In general, the canonical form of the SGS stress is

$$
\tau_{i j(L E S)}=\frac{2}{3} k_{S G S} \delta_{i j}-2 \nu_{S G S} S_{i j}+E A T_{i j}=E V M_{i j(L E S)}+E A T_{i j},
$$

where $E A T_{i j}$ is the extra anisotropic term (EAT). Note that for RANS, we often use the following expression for the Reynolds stress:

$$
\tau_{i j(R A N S)}=\frac{2}{3} k \delta_{i j}-2 \nu_{t} S_{i j}+H O T_{i j}=E V M_{i j(R A N S)}+H O T_{i j},
$$


where $k$ and $\nu_{t}$ are the turbulence energy and the eddy viscosity, respectively. In Eq. (16), $H O T_{i j}$ is a kind of EAT but is usually called the higher-order term (HOT) because this term consists of second- and/or third-order products of the strain-rate and vorticity tensors (see Appendix).

If we connect the LES and RANS regions according to Eq. (6), $\tau_{i j}$ is written as

$$
\begin{aligned}
\tau_{i j}=\left(1-f_{h b}\right) \tau_{i j(R A N S)}+f_{h b} & \tau_{i j(L E S)} \\
=\left(1-f_{h b}\right) E V M_{i j(R A N S)} & +f_{h b} E V M_{i j(L E S)} \\
+ & {\left[\left(1-f_{h b}\right) H O T_{i j}+f_{h b} E A T_{i j}\right] . }
\end{aligned}
$$

Finally, we obtain

$$
\tau_{i j}=\left(1-f_{h b}\right) E V M_{i j(R A N S)}+f_{h b} E V M_{i j(L E S)}+E A T_{i j(H L R)},
$$

where

$$
E A T_{i j(H L R)}=\left(1-f_{h b}\right) H O T_{i j}+f_{h b} E A T_{i j} .
$$

Note that if we take only the first two terms on the right-hand side of Eq. (18), $\tau_{i j}$ has a canonical form of isotropic EVM that has most often been adopted for other HLR models.

In considering Eq. (18) applied to Eq. (2), the divergence of the EAT

$$
-\frac{\partial E A T_{i j}(H L R)}{\partial x_{j}}
$$

can be regarded as an additional source term in the transport equation that directly influences the momentum along the $i$-direction. An important point should be noted that the effect of Eq. (20) originally derives from a physicsoriented discussion because the $E A T_{i j}$ included in the $E A T_{i j(H L R)}$ of Eq. (19) is based on the scale-similarity modeling concept[25].

\section{Test Cases and Computational Conditions}

The main purpose of this study is to explain why the present anisotropic HLR model improves the predictive performance in the LES-RANS interface region. To investigate this issue, we apply two types of HLR models to fullydeveloped plane channel flows with various grid resolutions. One is the original (full) version of the anisotropic HLR model as described in Eq. (18), and the other is its isotropic EVM version consisting of only the first two terms on the right-hand side of Eq. (18). In this study, the former full version is referred to as "HLR-EAT," while the latter as "HLR-NOEAT."

The computational parameters are summarized in Table 1. The Reynolds number $\operatorname{Re}_{\tau}\left(=u_{\tau} \delta / \nu\right)$ is set at 1000 , which is almost the same as the DNS by Abe et al.[27], i.e., $R e_{\tau}=1020$. In this study, three grid resolutions were chosen to investigate the relation between the HLR model and the "double-buffer 
Table 1 Computational parameters for channel-flow cases.

\begin{tabular}{|c|c|c|c|c|c|c|c|c|}
\hline Case & Grid numbers & \multicolumn{2}{|c|}{ Domain $(x-z)$} & $\Delta x$ & \multicolumn{3}{|c|}{$\overline{\Delta y}$} & $\Delta z$ \\
\hline C1E3A & $257 \times 181 \times 257$ & $6.4 \delta \times 3$ & & 0.025 & & $10^{-4}$ & 0.034 & 0.013 \\
\hline C1E3B & $65 \times 181 \times 65$ & $6.4 \delta \times 3$ & & 0.1 & & $10^{-4}$ & -0.034 & 0.05 \\
\hline C1E3C & $33 \times 181 \times 33$ & $6.4 \delta \times 3$ & & 0.2 & & $10^{-4}$ & -0.034 & 0.1 \\
\hline C1E3D & $257 \times 181 \times 257$ & $6.4 \delta \times 3$ & & 0.025 & & $10^{-4}$ & -0.034 & 0.013 \\
\hline C1E3E & $65 \times 181 \times 65$ & $6.4 \delta \times 3$ & & 0.1 & & $10^{-4}$ & -0.034 & 0.05 \\
\hline C1E3F & $33 \times 181 \times 33$ & $6.4 \delta \times 3$ & & 0.2 & & $10^{-4}$ & -0.034 & 0.1 \\
\hline Case & Model & $R e_{\tau}$ & $\Delta x^{+}$ & $\Delta ?$ & & $\Delta z^{+}$ & $\Delta$ & \\
\hline C1E3A & HLR-EAT & 1000 & 25 & 0.3 & & 13 & $2 \times 1$ & $j^{-4}$ \\
\hline C1E3B & HLR-EAT & 1000 & 100 & 0.3 & & 50 & $5 \times 1$ & $\rho^{-4}$ \\
\hline $\mathrm{C} 1 \mathrm{E} 3 \mathrm{C}$ & HLR-EAT & 1000 & 200 & 0.3 & & 100 & $1 \times$ & $y^{-3}$ \\
\hline C1E3D & HLR-NOEAT & 1000 & 25 & 0.3 & & 13 & $2 \times$ & $0^{-4}$ \\
\hline C1E3E & HLR-NOEAT & 1000 & 100 & 0.3 & & 50 & $5 \times$ & $0^{-4}$ \\
\hline C1E3F & HLR-NOEAT & 1000 & 200 & 0.3 & & 100 & $1 \times$ & $0^{-3}$ \\
\hline
\end{tabular}

problem." First, to confirm the basic performance of the present simulation, we adopted a sufficiently fine grid resolution, in which the computational domain (i.e., $6.4 \delta \times 2 \delta \times 3.2 \delta$ ) is covered by grid nodes consisting of $257 \times 181 \times 257$ in the streamwise $(x)$, wall-normal $(y)$ and spanwise $(z)$ directions, respectively. In this case, the grid resolutions in the $x$ and $z$ directions become $\Delta x^{+}=$ 25 and $\Delta y^{+}=13$, respectively, which are fine enough for LES. The other two cases adopt much coarser grid resolutions with the same grid spacing maintained in the wall-normal direction (see Table 1). In fact, the coarsest grid resolution of $33 \times 181 \times 33$ provides $\Delta x^{+}=200$ and $\Delta z^{+}=100$, which generally is thought never to be applicable in an LES.

We perform calculations using an unstructured finite-volume procedure nearly identical to that of Muto et al.[28], where a vertex-centered type of storage is used on a grid. The second-order central difference scheme is used to discretize the spatial derivatives except for the convection term of the turbulence energy, which is discretized by the second-order upwind scheme. The time marching is based on the fractional step method[29], in which the secondorder Crank-Nicolson scheme is used for the velocity equations. Note that the first-order Euler implicit scheme is used for the transport equation of the turbulence energy. The coupling of the velocity and pressure fields is based on the simplified marker and cell method[30]. The flow rate on the controlvolume surface is estimated using the Rhie-Chow interpolation[31]. For the boundary conditions, the periodic condition is imposed in the streamwise and spanwise directions, whereas the no-slip conditions are specified at the wall surfaces. Note that in previous studies[20][22], the effects of a first-order timemarching scheme and a second-order upwind scheme adopted for the transport equation of turbulence energy were carefully investigated. These investigations confirmed that the time-integration and space-discretization schemes used in the transport equation of the turbulence energy did not have any crucial ef- 
Table 2 Comparison of the bulk-mean velocity $U_{b}$ and the skin friction coefficient $C_{f}$ with the DNS data[27].

\begin{tabular}{lcccc}
\hline \hline Case & $U_{b}$ & Error (\%) & $C_{f}$ & Error (\%) \\
\hline DNS[27] & 20.23 & & $4.89 \times 10^{-3}$ & \\
C1E3A & 19.70 & -2.63 & $5.15 \times 10^{-3}$ & 5.48 \\
C1E3B & 19.34 & -4.40 & $5.35 \times 10^{-3}$ & 9.41 \\
C1E3C & 20.29 & 0.27 & $4.86 \times 10^{-3}$ & -0.54 \\
C1E3D & 21.01 & 3.84 & $4.53 \times 10^{-3}$ & -7.27 \\
C1E3E & 21.20 & 4.82 & $4.45 \times 10^{-3}$ & -8.98 \\
C1E3F & 22.00 & 8.77 & $4.13 \times 10^{-3}$ & -15.48 \\
\hline \hline
\end{tabular}

fect on the final conclusions. Furthermore, some additional computations were also performed in this study to investigate how grid spacing and numerical viscosity influence the "double-buffer problem." The computational results are summarized in Appendix.

\section{Results and Discussion}

\subsection{Basic performance of the present HLR model}

First, to access the basic performance of the HLR models applied, the meanvelocity distributions are compared with the DNS data[27] in Fig. 1. Note that the distributions of the switching function $f_{h b}$ are also included in the figure. As seen in Fig. 1 (a), the present anisotropy HLR model (HLR-EAT) provides generally reasonable predictions for all the grid-resolution cases. We also confirm that a well-resolved full LES is achieved for the fine-grid-resolution case $(\mathrm{C} 1 \mathrm{E} 3 \mathrm{~A})$ because the switching function $f_{h b}$ is unity for the entire flow region (red line). More importantly, the "double-buffer problem" is not seen in the mean-velocity distributions, at least, for these three test cases. In contrast, from Fig. 1 (b), the predictions obtained using the isotropic EVM (HLRNOEAT) clearly show the "double-buffer problem" in the interface region, where steeper mean-velocity gradient is seen. As these two HLR models adopt the same form of EVM part, this difference in the mean-velocity predictions is thought to be caused by the EAT that has been introduced.

For a more detailed discussion, the error in the prediction of the skin friction coefficient $C_{f}$ is investigated. Following Piomelli et al.[18], the error is defined as

$$
\text { Error }(\%)=\frac{C_{f}-C_{f, D N S}}{C_{f, D N S}} \times 100, \quad C_{f}=\frac{\tau_{w}}{\rho U_{b}^{2} / 2},
$$

where $\tau_{w}$ and $U_{b}$ denote the mean wall stress and the bulk-mean velocity, respectively. The predicted values are compared with the DNS data in Table 2. Concerning the HLR-EAT cases (C1E3A-C1E3C), the error of $U_{b}$ is confined to within $\pm 5 \%$ and hence the full dispersion in the $C_{f}$ predictions for all grid resolutions has error of about $10 \%$. From an engineering viewpoint, this level 


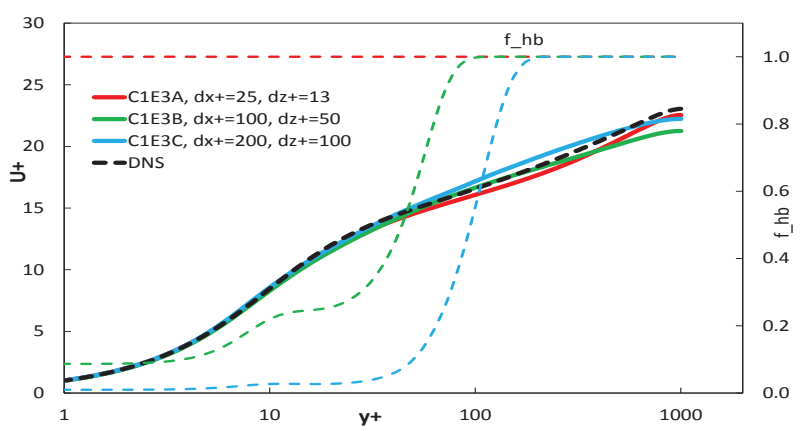

(a) The present model (HLR-EAT)

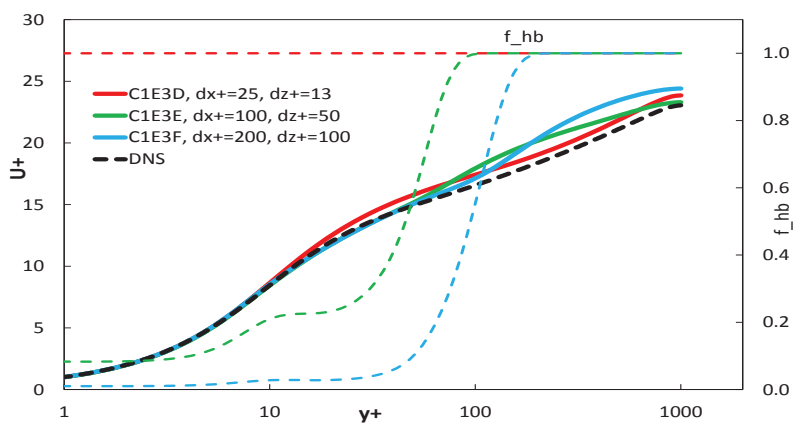

(b) Isotropic model (HLR-NOEAT)

Fig. 1 Comparison of the mean-velocity distributions for various grid resolutions.

is thought to be acceptable. Nevertheless, the error of HLR-NOEAT (C1E3DC1E3F) becomes larger than that of HLR-EAT, particularly for the coarsest grid case. This is caused mainly by the "double-buffer problem," as seen in Fig. 1 (b).

Figure 2 shows the distributions of the total $R e$-shear stress and turbulence energy predicted by the HLR-EAT, whereas those obtained by the HLRNOEAT are compared in Fig. 3. Note that the distributions include the contribution of the modeled part as well as the resolved part. As seen in Fig. 2, the $R e$-shear stress predicted by the HLR-EAT corresponds well to that obtained by the DNS. As for turbulence energy, the HLR-EAT also provides reasonable predictions in general, although some overpredictions are still seen in the near-wall region. In contrast, the HLR-NOEAT returns much poorer predictions for the turbulence energy as considerable overpredictions are seen in the interface region as the grid resolution becomes coarser. In regard to the $R e$-shear stress, although both models seem to generally give reasonable results, a careful investigation shows that non-negligible differences are seen in the distributions between the HLR-EAT and the HLR-NOEAT. This issue 


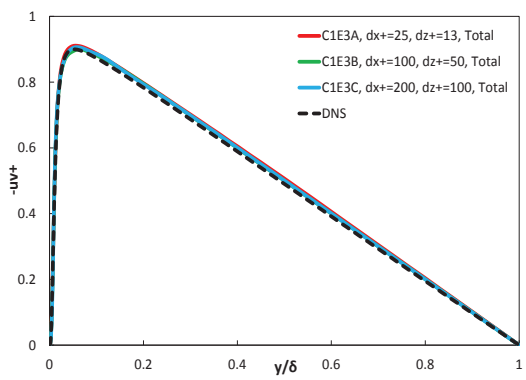

(a) Reynolds shear stress

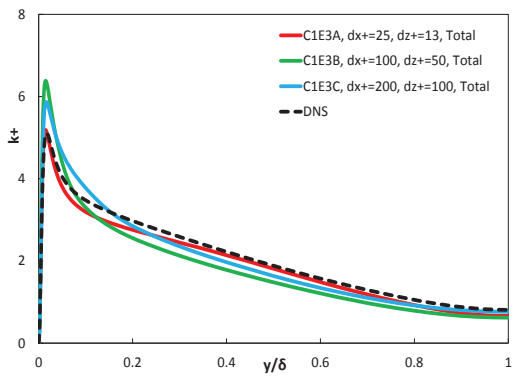

(b) Turbulence energy

Fig. 2 Distributions of the total Re-shear stress and turbulence energy predicted by the present model (HLR-EAT).

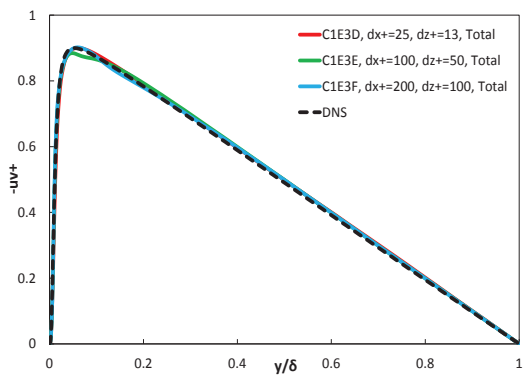

(a) Reynolds shear stress

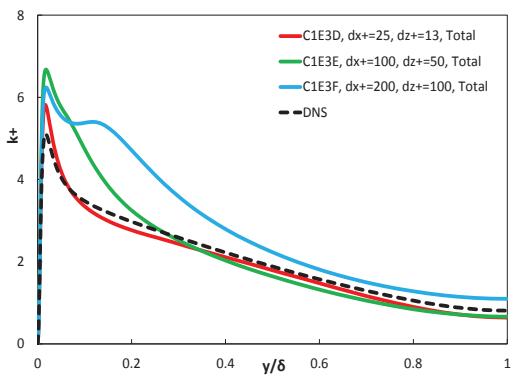

(b) Turbulence energy

Fig. 3 Distributions of the total Re-shear stress and turbulence energy predicted by the isotropic model (HLR-NOEAT).

will be discussed in more detail in the next section because the prediction of the $R e$-shear stress must be closely related to the mean-velocity prediction.

4.2 Effect of the extra anisotropic term in the LES-RANS interface region

In this section, we shall investigate the contribution that the EAT makes in improving the "double-buffer problem." Figure 4 shows the distributions of the total $R e$-shear stress that are enlarged in the horizontal direction. A careful look at Fig. 4 (b) shows that there are non-negligible differences among the distributions of HLR-NOEAT, where some underestimations are obtained in the interface regions at $y / \delta=0.05-0.1\left(y^{+}=50-100\right)$ for C1E3E and at $y / \delta=0.1-0.2\left(y^{+}=100-200\right)$ for $\mathrm{C} 1 \mathrm{E} 3 \mathrm{~F}$, respectively. As this feature corresponds directly to the mean-velocity prediction, more detailed discussion on the role of each part (i.e., modeled or resolved) is important. Furthermore, it should be made clear how the EAT works to improve the distribution of the $R e$-shear stress as seen in Fig. 4 (a).

To access the EAT contribution to the $R e$-shear stress, the distribution of $E A T_{i j_{(H L R)}}$ in Eq. (19) for HLR-EAT is compared with that of the modeled 


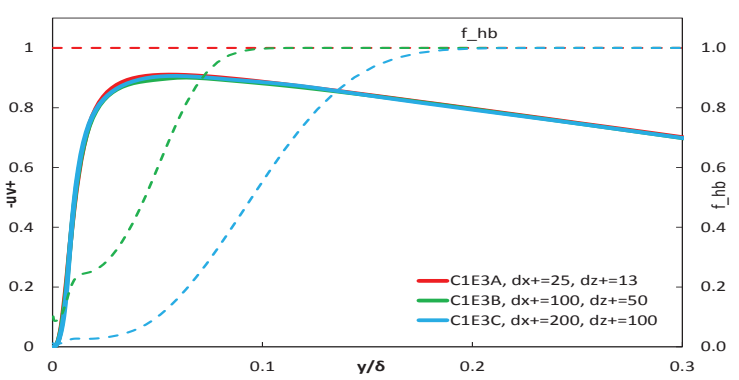

(a) HLR-EAT

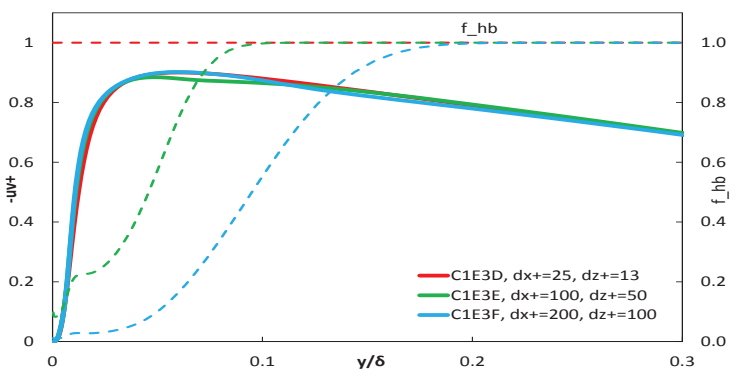

(b) HLR-NOEAT

Fig. 4 Comparison of the total Re-shear stress in the switching region.

stress $\tau_{i j}$ in Eq. (18) as well as the resolved stress and total stress in Fig. 5. From Fig. 4 (a), a full LES is achieved for C1E3A, where the grid resolution is fine enough. Although the EAT covers a considerable portion of the modeled part even for this test case, its contribution is not so significant. Nevertheless, as the grid resolution becomes coarser, the modeled part becomes more and more important. From Fig. 4 (b) and (c), the near-wall region is covered by the modeled stress (solid green lines). Although this contribution mainly derives from the RANS part introduced in the HLR model, it is also interesting that the effect of EAT appears particularly in the interface region (broken black lines). Because the EAT provides statistically the same sign as the other terms, this effect tends to increase the $R e$-shear stress.

Concerning the improvement of the HLR model in the interface region, the stochastic backscatter model[18] may be a promising way, in which a random source term is directly imposed in the Navier-Stokes equation. Interestingly, the stochastic backscatter model improves the "double-buffer problem" with no statistical effect on momentum transfer. This feature is different from that of the present anisotropic HLR model because the present model produces an effect on momentum transfer (see Fig. 5), with no energy transfer between the GS and SGS parts[20][22]. However, these two models have an important common feature in that they definitely increase the vorticity and enhance the vortex motions[18][24]. Consequently, an important key aspect in reducing 
the "double-buffer problem" is how we can successfully enhance the turbulent vortex structures in the interface region. One notes that, although the specification of the parameters in the stochastic backscatter model is arbitrary and ambiguous, the present EAT originally derives from a physics-oriented analysis on the scale-similarity modeling concept[25].

On the other hand, Rajamani and Kim[32] provided an encouraging idea that originally derived from a sophisticated discussion on a hybrid filter approach by Germano[33]. They proposed several additional terms to improve the model performance, particularly in the interface region. One of their additional terms for the modeled $R e$-shear stress has a formulation similar to that of the present EAT, although the filtering operator used has a slightly different meaning in processing the velocity-field data. This fact may also be related to why the present EAT works to enhance vortex structures in the interface region. 


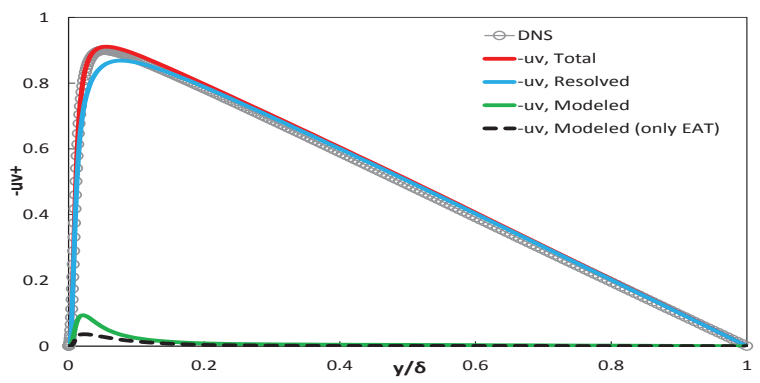

(a) C1E3A (fine grid):

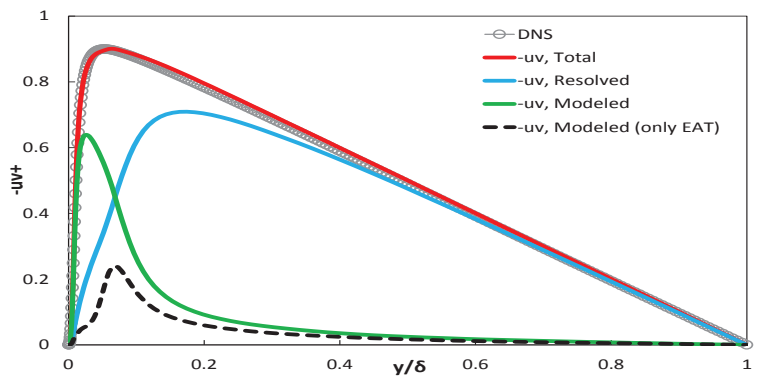

(b) C1E3B (medium grid)

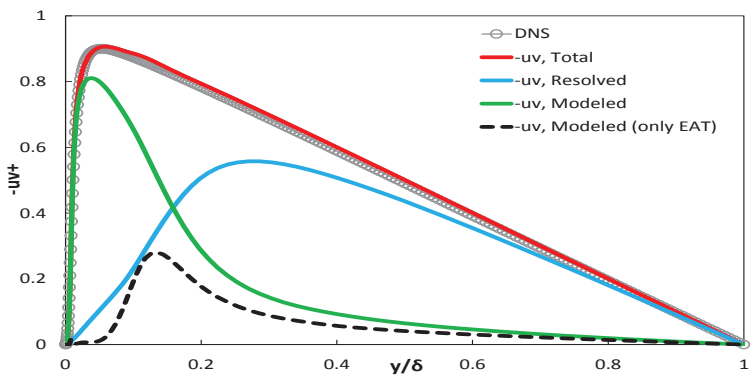

(c) C1E3C (coarse grid)

Fig. 5 Contribution of the EAT in modeled Re-shear stress (broken black lines show only the EAT effect, whereas solid green lines show the modeled stress). 


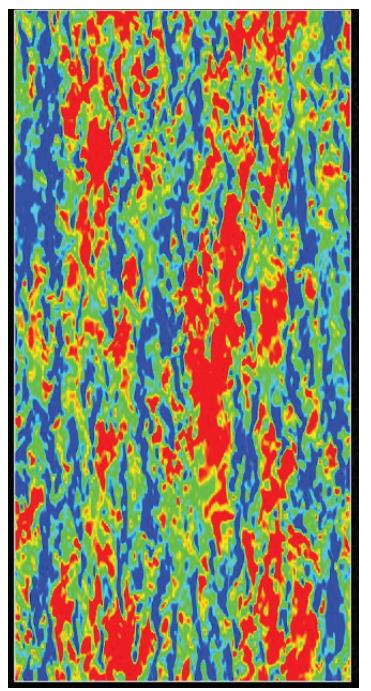

(a) $y^{+}=70(y / \delta=0.07)$

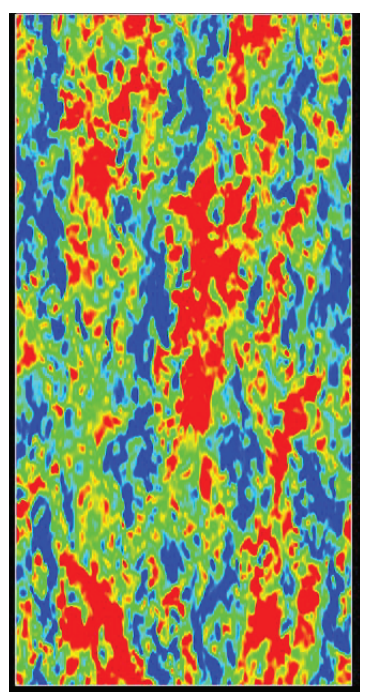

(b) $y^{+}=150(y / \delta=0.15)$

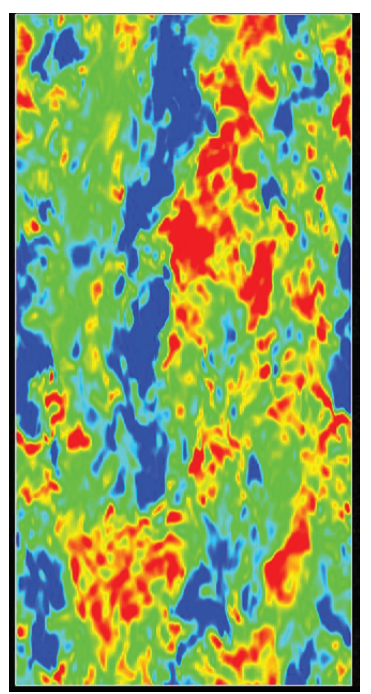

(c) $y^{+}=500(y / \delta=0.5)$

Fig. 6 Distributions of the streamwise velocity fluctuation at several $y$ locations for the fine grid-resolution case (C1E3A) (color ranges from -2 (blue) to 2 (red), normalized in wall units).

\subsection{Detailed investigation of the turbulent structures}

As seen in Fig. 5, the present EAT works to increase the Re-shear stress in a statistical manner. However, we cannot understand sufficiently just from statistics alone how the EAT affects unsteady turbulent structures. Therefore, in this section, we shall compare the distributions of the instantaneous streamwise velocity fluctuation in the interface region. In addition, to evaluate the size of the vortex structures, we also calculated the spanwise two-point correlation coefficients of the streamwise velocity fluctuation.

First, Fig. 6 shows the distributions of the streamwise velocity fluctuation at several $y$ locations for the fine grid-resolution case (C1E3A). Note that the location of $y^{+}=70(y / \delta=0.07)$ corresponds to the interface region for the medium grid-resolution case (C1E3B, C1E3E), and similarly the location of $y^{+}=150(y / \delta=0.15)$ for the coarse grid-resolution case (C1E3C, C1E3F). Note that the far-field location of $y^{+}=500(y / \delta=0.5)$ is selected for comparison because it is always involved in the LES region. From Fig. 6 (a) and (b), we find many small-scale turbulent structures as well as larger flow patterns colored in red (high-speed) or blue (low-speed). Note that, as found from Fig. 1, these locations of $y^{+}=70$ and 150 are generally involved in the logarithmic region of the mean-velocity distribution. Nevertheless, as seen in Fig. 6 (c), small-scale fluctuations tend to decrease, whereas larger flow patterns remain at the far-field location. 


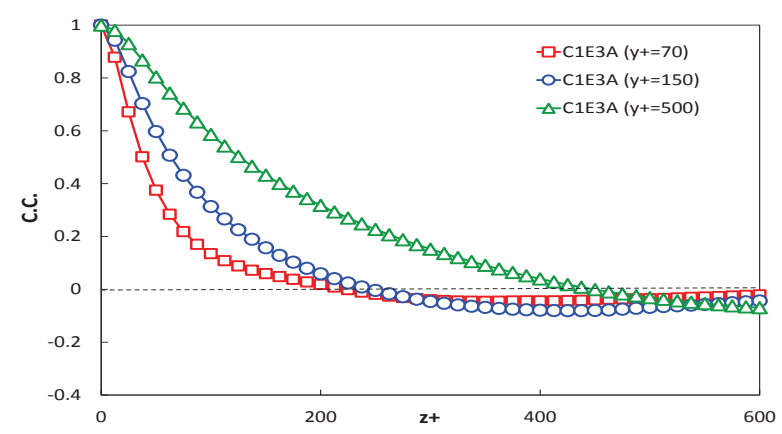

Fig. 7 Distributions of the spanwise two-point correlation coefficients of the streamwise velocity fluctuation for the fine grid-resolution case (C1E3A).

This fact is also confirmed from a comparison of the spanwise two-point correlation coefficients of the streamwise velocity fluctuation, as seen in Fig. 7. Note that these data are calculated for C1E3A at the same $y$ locations in Fig. 6. The understanding is that the size of the turbulent structures becomes smaller as the $y$ location becomes closer to the wall surface. This corresponds well to accepted beliefs and does not conflict with the insight garnered from Fig. 6. Therefore, the present results are thought to be useful for investigating unsteady turbulent motions.

We shall now compare the distributions of the velocity fluctuation obtained using C1E3B, C1E3C, C1E3E, and C1E3F with those using C1E3A. Note that in comparison, the results of $\mathrm{C} 1 \mathrm{E} 3 \mathrm{~A}$ are test-filtered and then reduced, using the filter width and the grid nodes that are the same as those of the corresponding grid-resolution case. If the computational results for coarser gridresolution cases are reasonably predicted, turbulent structures are expected to show characteristics similar to those of the test-filtered C1E3A data.

Figures 8 and 9 compare the results for the streamwise velocity fluctuation at $y^{+}=70(y / \delta=0.07)$ and $y^{+}=500(y / \delta=0.5)$, respectively, for the medium grid-resolution cases (C1E3B and C1E3E). Note that from Fig. 5 (b), the location of $y / \delta=0.07$ for this grid resolution is thought to be involved in the interface region, where the EAT works actively. As found from Fig. 8 (b), the results by HLR-EAT show small-scale turbulent structures, the sizes of which are similar to those of the test-filtered C1E3A data in Fig. 8 (a). However, HLR-NOEAT fails to reproduce this trend. From Fig. 8 (c), the vortex structures are larger than those in Fig. 8 (a) and small scale fluctuations seem to disappear. Figure 8 (c) may indicate a week effect of "super-streak structures" appearing in the interface region. This is thought to be closely related to the problem as seen in the mean-velocity distribution (see Fig. 1 (b)). On the other hand, looking at Fig. 9 (b) and (c), both HLR-EAT and HLRNOEAT show a similar trend at $y / \delta=0.5$, where large-scale flow patterns can be seen. Furthermore, their scales look similar to those of the test-filtered C1E3A data in Fig. 9 (a). 


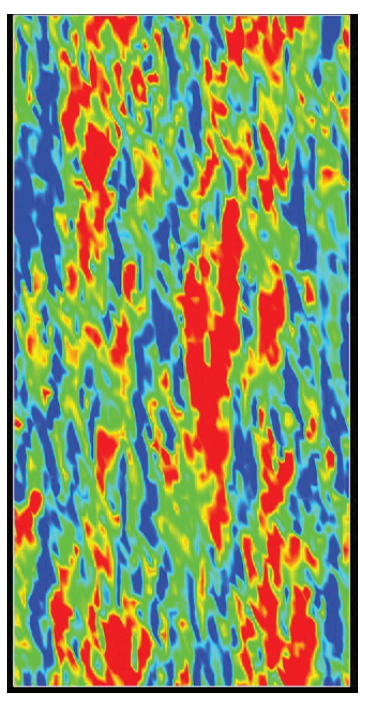

(a) C1E3A (test filtered)

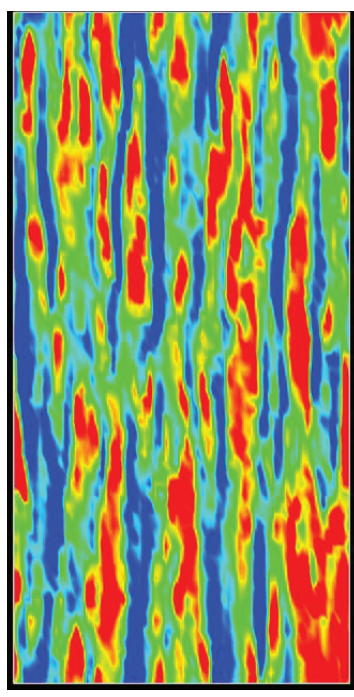

(b) C1E3B (HLR-EAT)

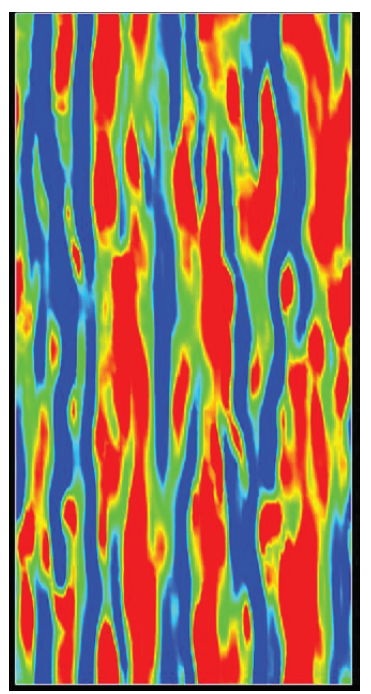

(c) C1E3E (HLR-NOEAT)

Fig. 8 Comparison of the streamwise velocity fluctuation at $y^{+}=70(y / \delta=0.07)$ for the medium grid-resolution case (color ranges from -2 (blue) to 2 (red), normalized in wall units). Note that the results of C1E3A are test-filtered and then reduced using the filter width and the grid nodes being the same as those of the medium grid case.

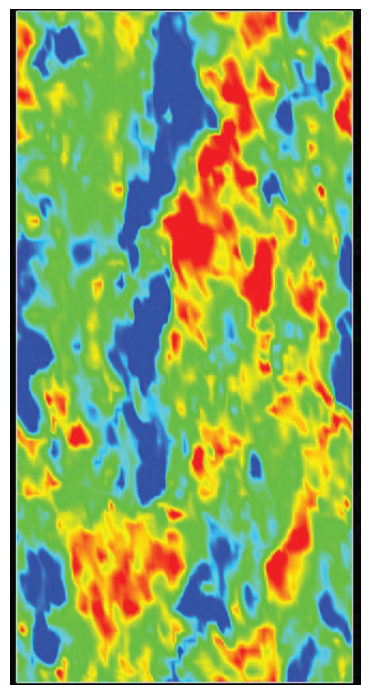

(a) C1E3A (test filtered)

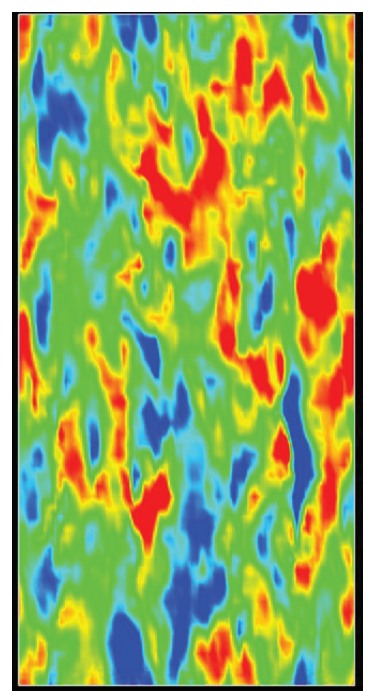

(b) C1E3B (HLR-EAT)

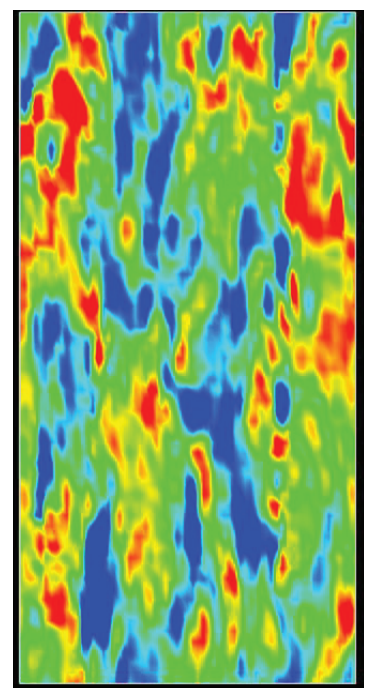

(c) C1E3E (HLR-NOEAT)

Fig. 9 As for Fig. 8 except for $y^{+}=500(y / \delta=0.5)$. 


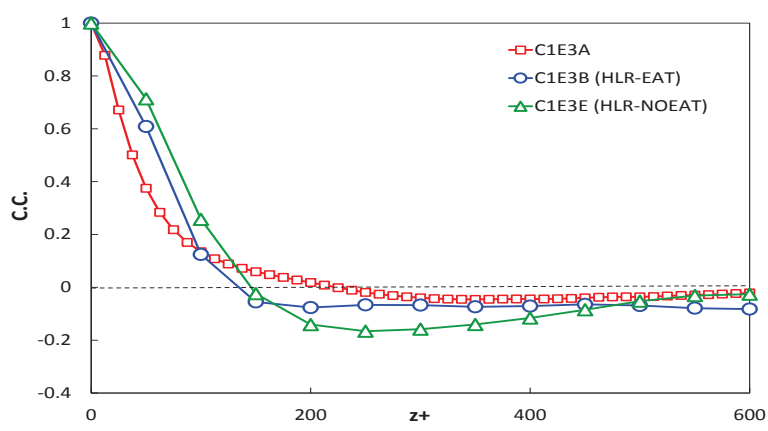

(a) $y^{+}=70(y / \delta=0.07)$

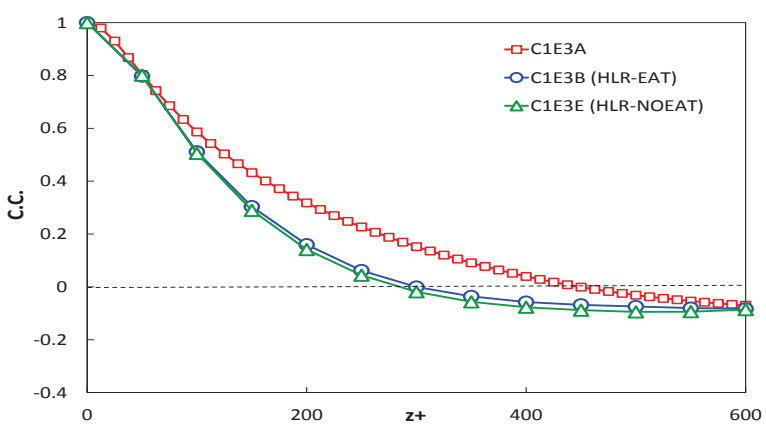

(b) $y^{+}=500(y / \delta=0.5)$

Fig. 10 Comparison of the spanwise two-point correlation coefficients of the streamwise velocity fluctuation for the medium grid-resolution case.

Figure 10 compares the spanwise two-point correlation coefficients of the streamwise velocity fluctuation for $\mathrm{C} 1 \mathrm{E} 3 \mathrm{~B}$ and $\mathrm{C} 1 \mathrm{E} 3 \mathrm{E}$ with those for the fine grid-resolution case (C1E3A). Note that the $y$ locations are the same as in Figs. 8 and 9. From Fig. 10 (a), the size of the structures of HLR-EAT at $y^{+}=70$ is smaller than that of HLR-NOEAT and closer to that for C1E3A. Conversely, from Fig. 10 (b), the size of the structures at $y^{+}=500$ becomes larger compared with that at $y^{+}=70$. Both HLR-EAT and HLR-NOEAT exhibit a very similar trend in that almost the same size of structures is obtained. The estimated correlation coefficients are close in value to C1E3A up to $z^{+} \sim 100$, although some underpredictions are still seen.

Figures 11 and 12 provide a comparison of the results for the streamwise velocity fluctuation at $y^{+}=150(y / \delta=0.15)$ and $y^{+}=500(y / \delta=0.5)$, respectively, for the coarse grid-resolution cases (C1E3C and C1E3F). Note that from Fig. 5 (c), the understanding is that the location of $y / \delta=0.15$ for this grid resolution is involved in the interface region. From Fig. 11 (b) and (c), the turbulent structures of HLR-EAT are much smaller than those of HLRNOEAT in the interface region. The structure scales of HLR-EAT are closer 


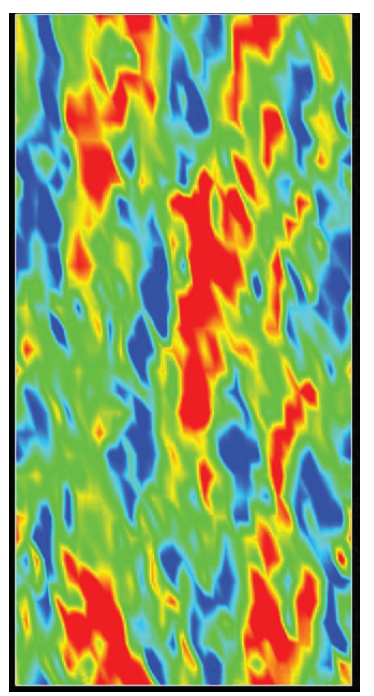

(a) C1E3A (test filtered)

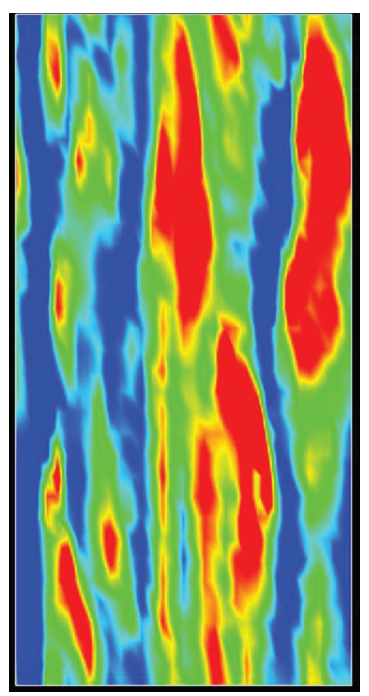

(b) C1E3C (HLR-EAT)

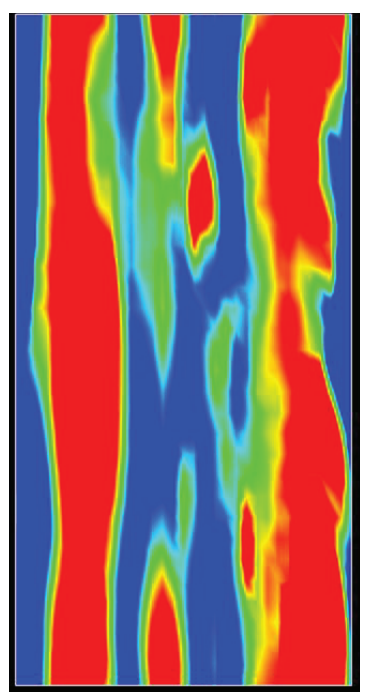

(c) C1E3F (HLR-NOEAT)

Fig. 11 Comparison of the streamwise velocity fluctuation at $y^{+}=150(y / \delta=0.15)$ for the coarse grid-resolution case (color ranges from -2 (blue) to 2 (red), normalized in wall units). Note that the results of $\mathrm{C} 1 \mathrm{E} 3 \mathrm{~A}$ are test-filtered and then reduced using the filter width and the grid nodes being the same as those of the coarse grid case.

to those of the test-filtered C1E3A data in Fig. 11 (a) compared with those of HLR-NOEAT. In Fig. 11 (c), large scale "super-streak structures" are clearly visible. In contrast, in Fig. 12 (b) and (c), both HLR-EAT and HLR-NOEAT show a similar trend at $y / \delta=0.5$, where large-scale flow patterns are evident. Their scales look similar to those of the test-filtered C1E3A data in Fig. 12 (a). Although small-scale fluctuations seem to disappear even for the HLR-EAT in Figs. 11 and 12, the results are thought to be acceptable by considering the coarse grid resolution in the streamwise and spanwise directions (i.e., $33 \times 33$ ), respectively.

The spanwise two-point correlation coefficients of the streamwise velocity fluctuation for C1E3C and C1E3F are compared with those for the fine gridresolution case (C1E3A) in Fig. 13, where the $y$ locations are the same as in Figs. 11 and 12. We glean from Fig. 13 (a) that the size of structures of HLREAT at $y^{+}=150$ is smaller than that of HLR-NOEAT and closer to that for C1E3A. In contrast, from Fig. 13 (b), the estimated correlation coefficients at $y^{+}=500$ for both HLR-EAT and HLR-NOEAT are close to C1E3A up to $z^{+} \sim 100$, whereas some underpredictions are seen in the results of HLR-EAT. Regarding this issue, a more detailed analysis is needed.

From the foregoing investigation, we found that the trend visible in Fig. 11 coincides with that in Fig. 8. As both these locations are involved in the LESRANS interface region, this behavior indicates that the EAT introduced in the 


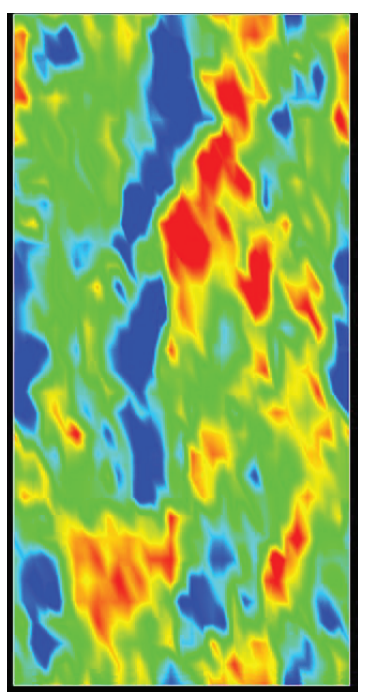

(a) C1E3A (test filtered)

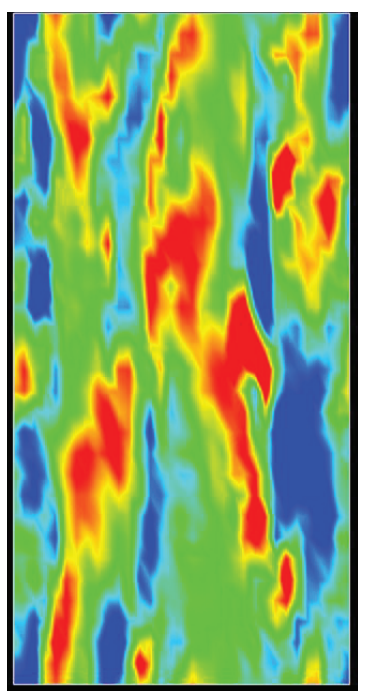

(b) C1E3C (HLR-EAT)

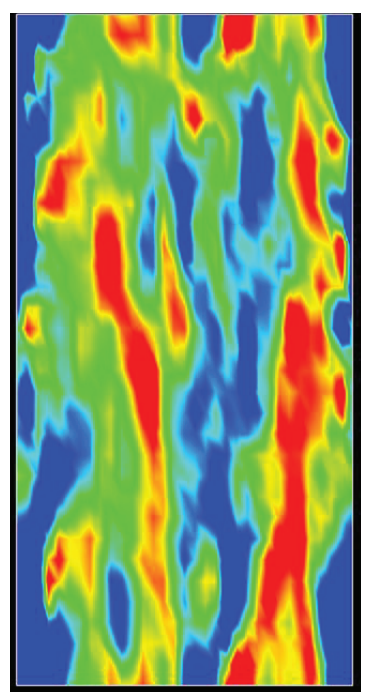

(c) C1E3F (HLR-NOEAT)

Fig. 12 As for Fig. 11 except for $y^{+}=500(y / \delta=0.5)$.

anisotropy-resolving SGS model works to prevent "super-streak structures" from being generated and thus reasonable scales of turbulent structures can be maintained. Indeed, this relates closely to the present HLR model being successful in reducing the "double-buffer problem" in the interface region. Conversely, turbulent structures tend to become larger in the region far from the wall surface.

Finally, to investigate the influence of turbulent fluctuations on the statistical values, we compare the distributions of the production and pressure-strain terms in the budget of the streamwise and shear components of the resolved $R e$-stress tensor as these two terms are the main contributors to the gain and loss parts, respectively. Figure 14 shows a comparison of these two terms for the resolved streamwise $R e$-normal stress; those of the $R e$-shear stress are compared in Fig. 15. Note that the distributions of the switching function $f_{h b}$ are also included in the figures for reference. As the point of discussion of the present study is model performance in the LES-RANS interface region, we focus mainly on the behavior in the region where $f_{h b}$ is changing from 0 to 1. A large deference is seen between HLR-EAT and HLR-NOEAT in Fig. 14, particularly in the distributions of the production term. The HLR-NOEAT for $\mathrm{C} 1 \mathrm{E} 3 \mathrm{E}$ and $\mathrm{C} 1 \mathrm{E} 3 \mathrm{~F}$ clearly returns local peak values in the LES-RANS interface region. This is thought to be a reason why the HLR-NOEAT provides considerable overpredictions of the turbulence energy in the interface region for the coarser-grid-resolution cases (see Fig. 3). However, concerning the Reshear stress (Fig. 15), a difference is seen instead in the pressure-strain term for the coarser-grid-resolution cases. The HLR-EAT returns relatively larger 


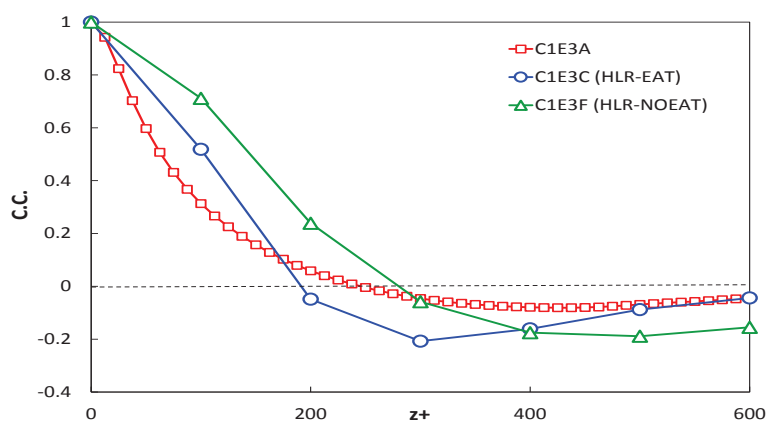

(a) $y^{+}=150(y / \delta=0.15)$

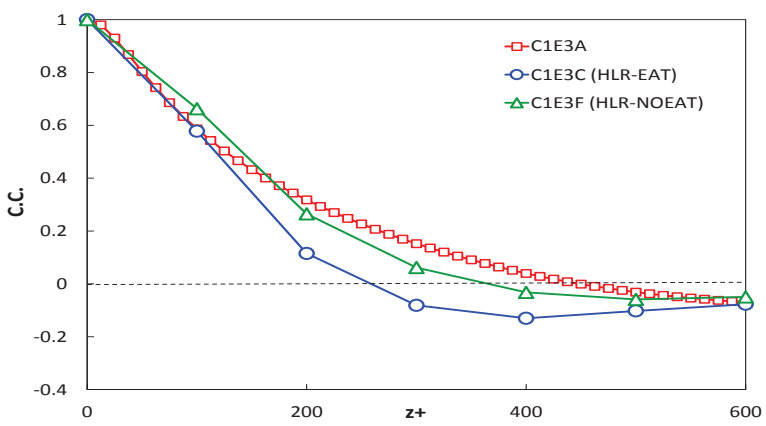

(b) $y^{+}=500(y / \delta=0.5)$

Fig. 13 Comparison of the spanwise two-point correlation coefficients of the streamwise velocity fluctuation for the coarse grid-resolution case.

values of the pressure-strain term in the interface region compared with those of the HLR-NOEAT. This increase may be a reason for the improvement in the prediction accuracy for the $R e$-shear stress (Fig. 2), although their relationship has not been made entirely clear. Further detailed analysis is needed.

\section{Concluding Remarks}

To explain why the anisotropy-resolving HLR model can reduce the "doublebuffer problem" in the LES-RANS interface region, we investigated the model performance at various grid resolutions by applying the model to plane channel flow. In particular, we focused on the role of the EAT introduced in the SGS model.

Familiarly, the "double-buffer problem" in the mean-velocity distribution arises mainly because the $R e$-shear stress is insufficient in the interface region. In regard to this issue, the EAT was found to give statistically increase to the Re-shear stress. This compensated the total Re-shear stress and thus a 


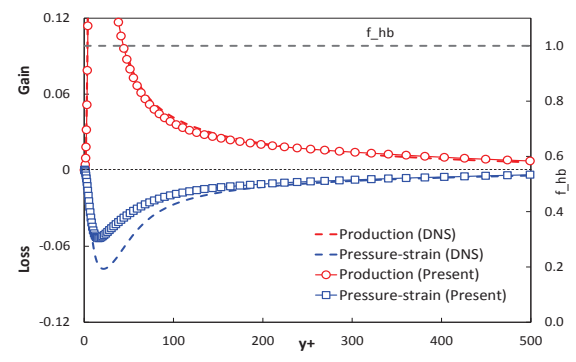

(a) C1E3A

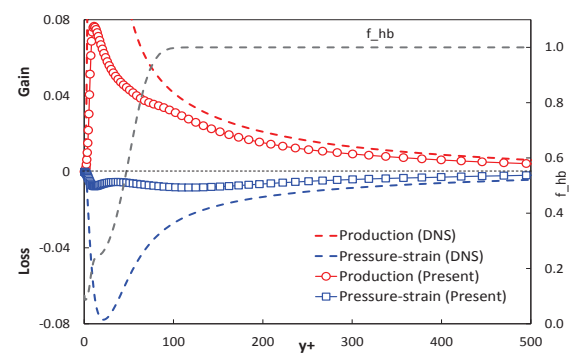

(b) C1E3B

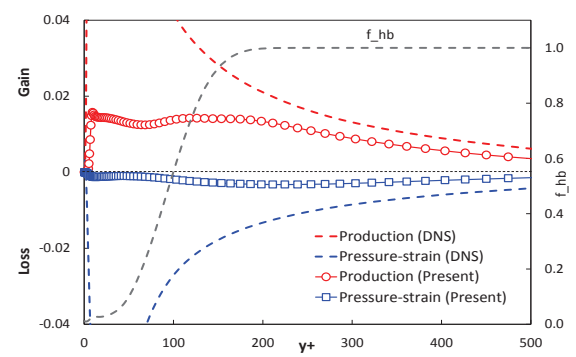

(c) $\mathrm{C} 1 \mathrm{E} 3 \mathrm{C}$

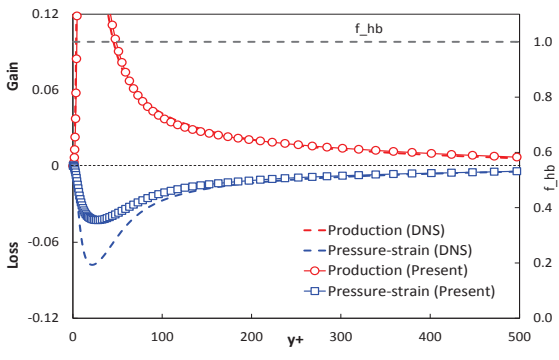

(d) C1E3D

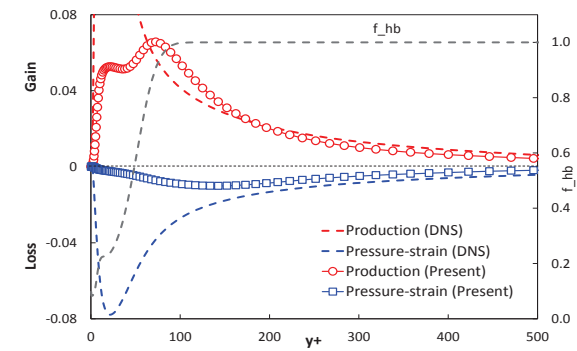

(e) C1E3E

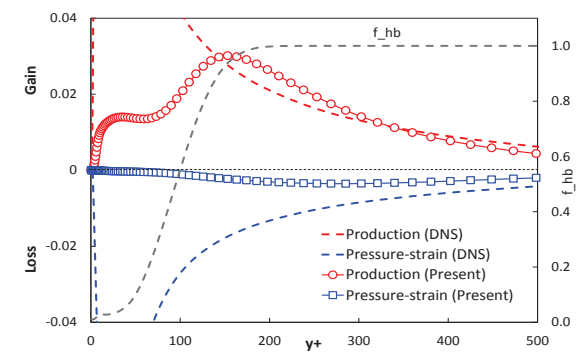

(f) $\mathrm{C} 1 \mathrm{E} 3 \mathrm{~F}$

Fig. 14 Comparison of the production (red) and pressure-strain (blue) terms in the budget of the resolved streamwise Re-normal stress (lines with symbols: the present simulation results, color dashed lines: DNS data taken from Lee and Moser[34], gray dashed lines: the present LES/RANS switching function).

considerable improvement in the mean-velocity prediction with no (or, at least, very small) "double-buffer problem" was achieved.

Furthermore, if the "double-buffer problem" occures, we often see "superstreak structures" in the interface region that look like low-speed streaks generated in the near-wall region. To investigate how the EAT affects turbulent structures in the interface region, the results for instantaneous velocity fluctuations obtained with and without the EAT were compared. The comparison indicated that the vortex structures were found to be divided into smaller sizes as an effect of the EAT and therefore "super-streak structures" tended to become weaker. This is a notable feature of the present HLR model. 


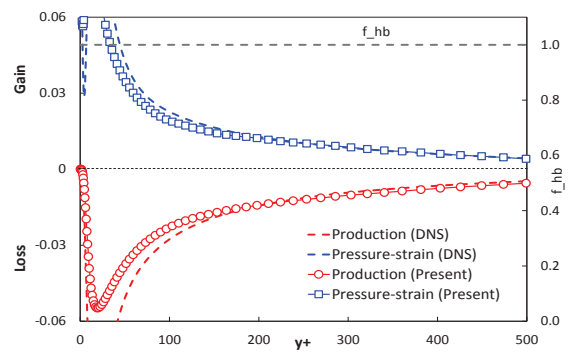

(a) $\mathrm{C} 1 \mathrm{E} 3 \mathrm{~A}$

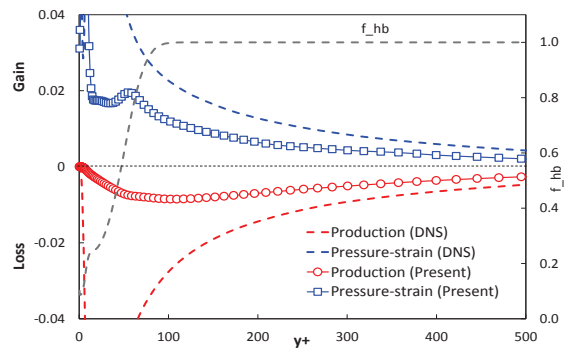

(b) C1E3B

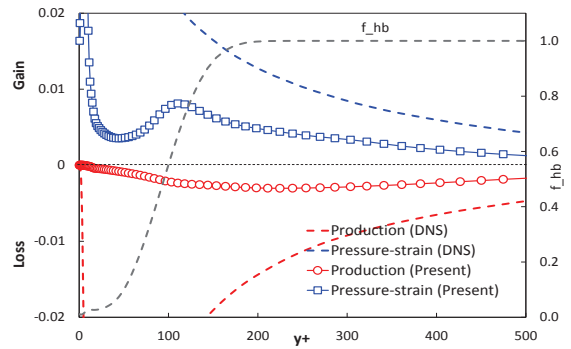

(c) $\mathrm{C} 1 \mathrm{E} 3 \mathrm{C}$

HLR-EAT

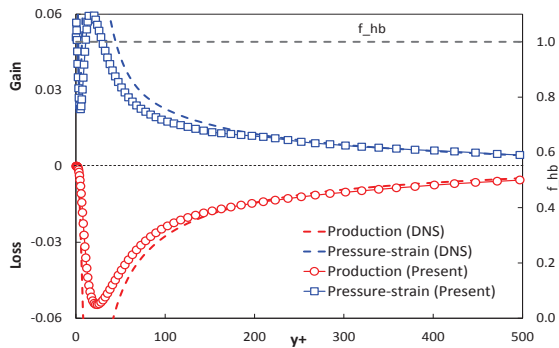

(d) C1E3D

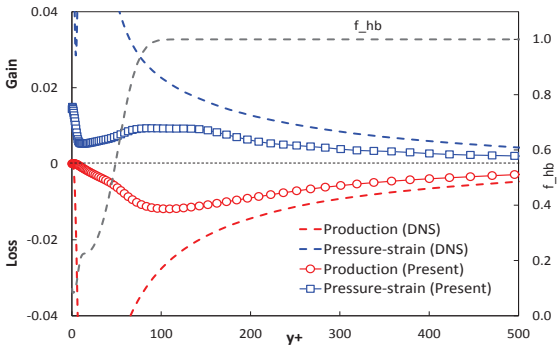

(e) C1E3E

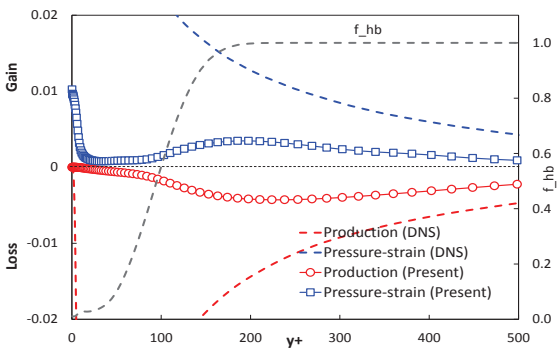

(f) $\mathrm{C} 1 \mathrm{E} 3 \mathrm{~F}$

HLR-NOEAT

Fig. 15 Comparison of the production (red) and pressure-strain (blue) terms in the budget of the resolved Re-shear stress (lines with symbols: the present simulation results, color dashed lines: DNS data taken from Lee and Moser[34], gray dashed lines: the present LES/RANS switching function).

From this fact, introducing this type of EAT into the LES part of an HLR model is considered to be a promising way to enhance vortex structures in the LES-RANS interface region, thereby resulting in a successful improvement of the "double-buffer problem." The findings obtained here indicate from an engineering viewpoint the basic potential of the present anisotropy-resolving HLR model. 
Acknowledgements This research was supported by JSPS KAKENHI Grant Number JP16K05042. This research was also supported by the "Advanced Computational Scientific Program" of the Research Institute for Information Technology, Kyushu University, Japan. The present computation was primarily carried out using the computer facilities at the Research Institute for Information Technology, Kyushu University, Japan.

\section{Appendix}

A.1 Detailed descriptions of turbulence models adopted in this study

In the following section, we briefly describe the one-equation NLEVM and the LES/RANS switching function used in the HLR model of Abe[19].

The Reynolds stress $\overline{u_{i} u_{j}}$ in the RANS region is modeled as follows:

$$
\begin{aligned}
\overline{u_{i} u_{j}}= & \frac{2}{3} k \delta_{i j}-2 k \tau C_{B}\left[1+\left\{1-f_{w}(26)\right\} f_{s 1}\right] S_{i j} \\
& +4 k \tau^{2} C_{D} C_{B}\left\{1-f_{w}(26)\right\}\left\{-\left(S_{i k} \Omega_{k j}-\Omega_{i k} S_{k j}\right)\right. \\
& \left.+\left(1-f_{s 2}\right)\left(S_{i k} S_{k j}-\frac{\delta_{i j}}{3} S^{2}\right)\right\} \\
& +2 k^{w} b_{i j},
\end{aligned}
$$

where $\Omega_{i j}$ is the vorticity tensor written as

$$
\Omega_{i j}=\frac{1}{2}\left(\frac{\partial \bar{U}_{i}}{\partial x_{j}}-\frac{\partial \bar{U}_{j}}{\partial x_{i}}\right) .
$$

In Eq. (22), the model function $f_{w}$ is defined by[35]

$$
f_{w}(\xi)=\exp \left\{-\left(\frac{n^{*}}{\xi}\right)^{2}\right\}
$$

where $\xi$ is a prescribed constant. In Eq. $(24), n^{*}\left(=(\nu \varepsilon)^{1 / 4} n / \nu\right)$ is the nondimensional wall distance with the Kolmogorov scale[36], where $n$ is uniquely determined as the shortest distance from all of the wall surfaces. In the model, the characteristic time scale $\tau$ and the model coefficients are given as[37]:

$$
\begin{gathered}
\tau=\frac{\nu_{t}}{k}, \quad \nu_{t}=C_{\mu} f_{\mu} \frac{k^{2}}{\varepsilon}, \quad f_{\mu}=\left[1+\frac{35}{R_{t}^{\frac{3}{4}}} \exp \left\{-\left(\frac{R_{t}}{30}\right)^{\frac{3}{4}}\right\}\right]\left\{1-f_{w}(26)\right\}, \\
C_{B}=\frac{1}{1+\frac{22}{3}\left(C_{D} \tau\right)^{2} \Omega^{2}+\frac{2}{3}\left(C_{D} \tau\right)^{2}\left(\Omega^{2}-S^{2}\right) f_{B}} \\
f_{s 1}=f_{r 1} f_{r 2} C_{s 1}\left(C_{D} \tau\right)^{2}\left(\Omega^{2}-S^{2}\right), \quad f_{s 2}=f_{r 1} f_{r 2}\left\{1+C_{s 2} C_{D} \tau(\Omega-S)\right\} \\
f_{B}=1+C_{\eta} C_{D} \tau(\Omega-S), \quad f_{r 1}=\frac{\Omega^{2}-S^{2}}{\Omega^{2}+S^{2}}, \quad f_{r 2}=\frac{S^{2}}{\Omega^{2}+S^{2}} \\
S^{2}=S_{m n} S_{m n}, \quad \Omega^{2}=\Omega_{m n} \Omega_{m n}, \quad S=\sqrt{S^{2}}, \quad \Omega=\sqrt{\Omega^{2}} \\
C_{D}=0.8, \quad C_{\mu}=0.12, \quad C_{\eta}=100, \quad C_{s 1}=0.15 C_{\eta}, \quad C_{s 2}=0.07 C_{\eta},
\end{gathered}
$$

where $R_{t}\left(=k^{2} / \nu \varepsilon\right)$ is the turbulent Reynolds number. 
In Eq. $(22),{ }^{w} b_{i j}$ is introduced to improve the predictive performance of the near-wall stress anisotropy[37], that is

$$
\begin{aligned}
{ }^{w} b_{i j}=f_{w}(26)\left[-\alpha_{w}\right. & \frac{1}{2}\left(d_{i} d_{j}-\frac{\delta_{i j}}{3} d_{k} d_{k}\right) \\
+\left(1-f_{r 1}{ }^{2}\right) \tau_{d}^{2}\{ & -\frac{\beta_{w} C_{w}}{1+C_{w} \tau_{d}^{2} \sqrt{S^{2} \Omega^{2}}}\left(S_{i k} \Omega_{k j}-\Omega_{i k} S_{k j}\right) \\
& \left.\left.+\frac{\gamma_{w} C_{w}}{1+C_{w} \tau_{d}^{2} S^{2}}\left(S_{i k} S_{k j}-\frac{\delta_{i j}}{3} S^{2}\right)\right\}\right],
\end{aligned}
$$

where

$$
d_{i}=\frac{N_{i}}{\sqrt{N_{k} N_{k}}}, \quad N_{i}=\frac{\partial n}{\partial x_{i}}, \quad \tau_{d}=\left\{1-f_{w}(15)\right\} \frac{k}{\varepsilon}+f_{w}(15) \delta_{w} \sqrt{\frac{\nu}{\varepsilon}} .
$$

In this study, the following combined model is used to represent the near-wall fragment[17]:

$$
\begin{gathered}
{ }^{w} b_{i j}=f_{\tau}{ }^{w 1} b_{i j}+\left(1-f_{\tau}\right){ }^{w 2} b_{i j}, \quad f_{\tau}=\exp \left[-3\left\{1-f_{w}(26)\right\} \frac{k}{\varepsilon} \sqrt{S^{2}}\right], \\
{ }^{w 1} b_{i j}=\left(\alpha_{w}=1, \quad \beta_{w}=\frac{1}{4}, \quad \gamma_{w}=1.5, \quad \delta_{w}=1.0, \quad C_{w}=0.5\right), \\
{ }^{w 2} b_{i j}=\left(\alpha_{w}=0, \quad \beta_{w}=\frac{13}{30}, \quad \gamma_{w}=0.6, \quad \delta_{w}=3.0, \quad C_{w}=1.0\right) .
\end{gathered}
$$

Further detailed descriptions are given in Abe[17] and Abe et al.[37].

In the study, the turbulence energy is determined from the typical form of the transport equation:

$$
\frac{\mathrm{D} k}{\mathrm{D} t}=\frac{\partial}{\partial x_{j}}\left\{\left(\nu+\frac{\nu_{t}}{\sigma_{k}}\right) \frac{\partial k}{\partial x_{j}}\right\}-\overline{u_{i} u_{j}} \frac{\partial \bar{U}_{i}}{\partial x_{j}}-\varepsilon,
$$

where $\sigma_{k}(=1.2)$ is the model constant. The dissipation rate $\varepsilon$ in this oneequation model is algebraically modeled as[19]

$$
\varepsilon=f_{e q} \frac{k^{3 / 2}}{C_{e q} y}+\frac{2 \nu k}{y^{2}}, \quad f_{e q}=1-\exp \left\{-\left(\frac{\sqrt{k} y}{50 \nu}\right)^{2}\right\}
$$

where $C_{e q}(=2.5)$ is the model constant and $f_{e q}$ is a damping function introduced to adjust the distribution of $\varepsilon$ in the near-wall region.

Abe[19] also proposed a new idea for connecting the LES and RANS regions smoothly. In this model, the switching function $f_{h b}$ in Eq. (5) adopted is

$$
f_{h b}=1-\exp \left\{-\left(C_{h b} \frac{l_{K L}}{\Delta}\right)^{3}\right\}
$$


where $C_{h b}(=30)$ is the model constant. In Eq. (31), a new length scale $l_{K L}$ is introduced instead of the simple wall distance $y$, that is given by

$$
l_{K L}=\frac{1}{C_{K L}^{3 / 2} f_{K L}} \frac{k^{3 / 2}}{\varepsilon}, \quad f_{K L}=1-\exp \left\{-\left(\frac{\sqrt{k} y}{C_{K L} \nu}\right)^{3 / 2}\right\},
$$

where $C_{K L}(=15)$ is the model constant and $f_{K L}$ is the model function to represent the characteristics of the near-wall turbulence. This model is originally based on the length scale of an energy-containing eddy with some modifications by introducing knowledge of the Kolmogorov microscale thought to be reasonable for representing near-wall turbulence. A notable feature of the present HLR model that distinguishes it from other previous models is in guaranteeing a full LES under a sufficiently fine grid-resolution condition. In fact, as the grid resolution becomes finer, $f_{h b}$ automatically approaches 1 for the whole flow field. A further detailed description of the present HLR model is given in Abe[19].

\section{A.2 Further validation of the present simulation}

As an HLR model generally uses grid-spacing details in its model expressions, investigating the model performance under different grid-spacing conditions is valuable. Therefore, for further validation of the present simulation, additional computations were performed using another type of grid spacing, .i.e., $\Delta x=$ $\Delta z$, which is often adopted in this field of research. Furthermore, to check the effect of the spanwise length of the computational domain on the prediction accuracy, an additional calculation was performed with a wider domain in the spanwise direction. The computational parameter settings are listed in Table 3. The mean-velocity distributions obtained are compared in Fig. 16, from which it is concluded that all the predictions correspond well to the DNS data and the "double-buffer problem" is not seen. This confirms that the present anisotropic HLR model works for other grid spacings. We also verify that the computational domain used suffices to provide reasonable simulation results.

Another concern is the effect of the convection term on the performance of the HLR models. In this study, the 2nd-order central difference scheme was adopted for the convection term in the momentum equation, and therefore no numerical viscosity was explicitly involved. However, for more practical engineering applications and/or very high-Re turbulent flows, we generally need numerical viscosities to establish stable computations. Therefore, we investigated the influence of this issue on the convection scheme on the primary aspect of this study. We applied two other types of convection schemes to the coarse grid-resolution cases, i.e., C1E3C and C1E3F. One was a combination of the 2nd-order central (95\%) and 1st-order upwind (5\%) difference schemes, whereas the other although similar has a blending factor of $90 \%$ (2nd) and 10\% (1st). The main purpose of these calculations was to 
Table 3 Computational parameters for additional channel-flow cases.

\begin{tabular}{lccccc}
\hline \hline Case & Grid numbers & Domain $(x-z)$ & $\Delta x$ & $\Delta y$ & $\Delta z$ \\
\hline C1E3G & $129 \times 181 \times 65$ & $6.4 \delta \times 3.2 \delta$ & 0.05 & $3 \times 10^{-4}-0.034$ & 0.05 \\
C1E3H & $65 \times 181 \times 33$ & $6.4 \delta \times 3.2 \delta$ & 0.1 & $3 \times 10^{-4}-0.034$ & 0.1 \\
C1E3I & $65 \times 181 \times 65$ & $6.4 \delta \times 6.4 \delta$ & 0.1 & $3 \times 10^{-4}-0.034$ & 0.1 \\
\hline \hline
\end{tabular}

\begin{tabular}{lcccccc}
\hline \hline Case & Model & $R e_{\tau}$ & $\Delta x^{+}$ & $\Delta y^{+}$ & $\Delta z^{+}$ & $\Delta t$ \\
\hline C1E3G & HLR-EAT & 1000 & 50 & $0.3-34$ & 50 & $5 \times 10^{-4}$ \\
C1E3H & HLR-EAT & 1000 & 100 & $0.3-34$ & 100 & $1 \times 10^{-3}$ \\
C1E3I & HLR-EAT & 1000 & 100 & $0.3-34$ & 100 & $1 \times 10^{-3}$ \\
\hline \hline
\end{tabular}

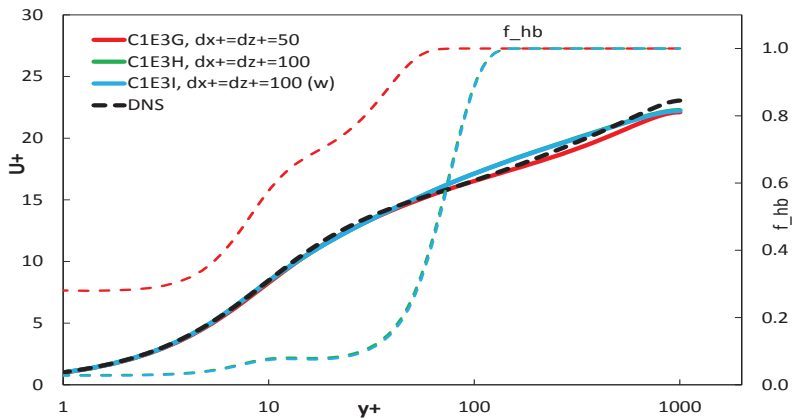

Fig. 16 Effect of another type of grid spacing, .i.e., $\Delta x=\Delta z$ on the mean-velocity predictions.

reveal how the convection scheme (in other words, the numerical viscosity involved) influences the essential features of the model performance in reducing the "double-buffer problem." The mean-velocity distributions obtained by the HLR-EAT are compared with those by the HLR-NOEAT in Fig. 17. The HLREAT provides in general reasonable predictions for all test cases. Although the performance tends to decrease gradually as numerical viscosity increases, the "double-buffer problem" is not seen in all test cases. In contrast, the HLRNOEAT clearly shows the "double-buffer problem" in its predictions. From this fact, the present EAT is expected to have an essential influence on turbulent vortex structures for reducing the "double-buffer problem," regardless of the convection scheme adopted in program.

\section{References}

1. Smagorinsky, J.: General circulation experiments with the primitive equations. I. The basic experiment. Mon. Weather Rev. 91, 99-164 (1963).

2. Germano, M., Piomelli, U., Moin, P., Cabot, W.H.: A dynamic subgrid-scale eddy viscosity model, Phys. Fluids A 3, 1760-1765 (1991).

3. Lilly, D.K.: A proposed modification of the Germano subgridscale closure method. Phys. Fluids A 4, 633-635 (1992). 


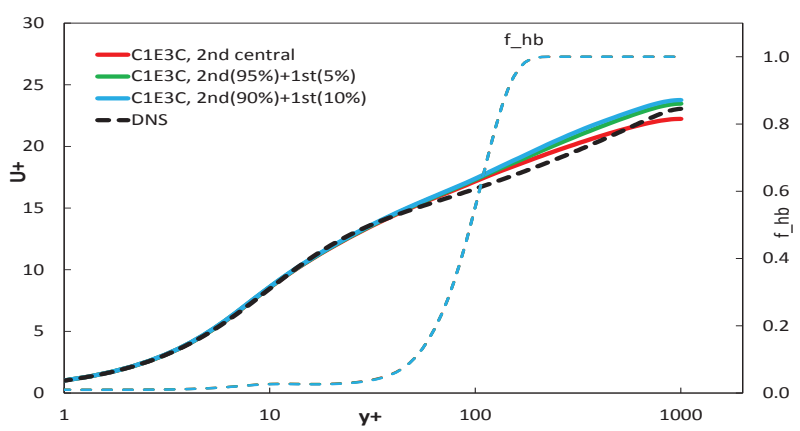

(a) The present model (HLR-EAT)

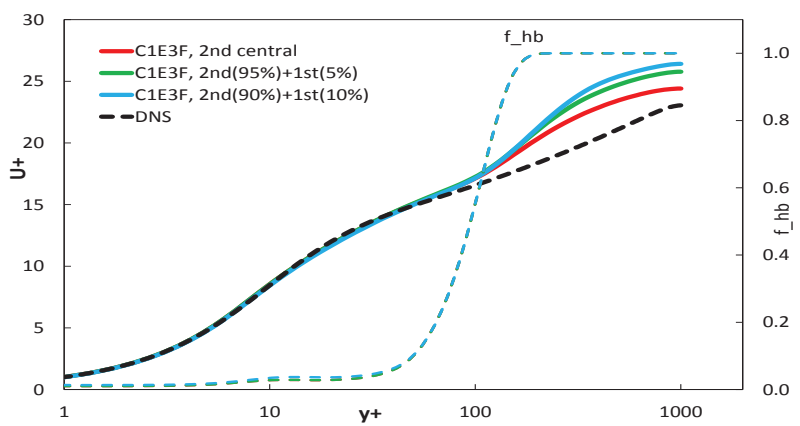

(b) Isotropic model (HLR-NOEAT)

Fig. 17 Effect of different convection schemes on model performance in regard to the "double-buffer problem."

4. Zang, Y., Street, R.L., Koseff, J.R.: A dynamic mixed subgrid-scale model and its application to turbulent recirculating flows, Phys. Fluids A 5, 3186-3196 (1993).

5. Horiuti, K.: A new dynamic two-parameter mixed model for large-eddy simulation, Phys. Fluids 9, 3443-3464 (1997)

6. Morinishi, Y., Vasilyev, O.V.: A recommended modification to the dynamic twoparameter mixed subgrid scale model for large eddy simulation of wall bounded turbulent flow, Phys. Fluids 13, 3400-3410 (2001).

7. Balaras, E., Benocci, C., Piomelli, U.: Two-layer approximate boundary conditions for large-eddy simulations. AIAA J. 34, 1111-1119 (1996).

8. Nikitin, N.V., Nicoud, F., Wasistho, B., Squires, K.D., Spalart, P.R.: An approach to wall modeling in large-eddy simulations. Phys. Fluids 12, 1629-1632 (2000).

9. Hamba, F.: An attempt to combine large eddy simulation with the $k-\varepsilon$ model in a channelflow calculation. Theoret. Comput. Fluid Dynamics 14, 323-336 (2001).

10. Davidson, L., Peng, S.H.: Hybrid LES-RANS modelling: a one-equation SGS model combined with a $k-\omega$ model for predicting recirculating flows. Int. J. Numer. Meth. Fluids 43, 1003-1018 (2003).

11. Batten, P., Goldberg, U., Chakravarthy, S.: Interfacing statistical turbulence closures with large-eddy simulation. AIAA J. 42, 485-492 (2004).

12. Hanjalic, K, Hadziabdic, M., Temmerman, L., Leschziner, M.A.: Merging LES and RANS strategies: zonal or seamless coupling? Direct and Large Eddy Simulation V (R. Friedrich et al. (eds)), Kluwer Academic Publ., 451-464 (2004). 
13. Temmerman, L., Hadziabdic, M., Leschziner, M.A., Hanjalic, K.: A hybrid two-layer URANS-LES approach for large eddy simulation at high Reynolds numbers. Int. J. Heat and Fluid Flow 26, 173-190 (2005).

14. Spalart, P.R., Deck, S., Shur, M.L., Squires, K.D., Strelets, M.Kh., Travin, A.: A new version of detached-eddy simulation, resistant to ambiguous grid densities. Theoret. Comput. Fluid Dynamics 20, 181-195 (2006).

15. Breuer, M., Jaffrezic, B., Arora, K.: Hybrid LES/RANS technique based on a oneequation near-wall model, Theoret. Comput. Fluid Dynamics 22, 157-187 (2008).

16. Deck, S.: Recent improvements in the Zonal Detached Eddy Simulation (ZDES) formulation. Theoret. Comput. Fluid Dynamics 26, 523-550 (2012).

17. Abe, K.: A hybrid LES/RANS approach using an anisotropy-resolving algebraic turbulence model, Int. J. Heat Fluid Flow 26, 204-222 (2005).

18. Piomelli, U., Balaras, E., Pasinato, H., Squaires, K.D., Spalart, P.R.: The inner-outer layer interface in large-eddy simulations with wall-layer models. Int. J. Heat and Fluid Flow 24, 538-550 (2003).

19. Abe, K.: An advanced switching parameter for a hybrid LES/RANS model considering the characteristics of near-wall turbulent length scales, Theoretical and Computational Fluid Dynamics 28, 499-519 (DOI: 10.1007/s00162-014-0328-3) (2014).

20. Abe, K.: An improved anisotropy-resolving subgrid-scale model with the aid of a scalesimilarity modeling concept, Int. J. Heat Fluid Flow 39, 42-52 (2013).

21. Uruma, O., Kihara, H., Abe, K.: An improvement of hybrid LES/RANS model for complex turbulence with the aide of an anisotropy-resolving subgrid-scale Model. Proceedings of the 29th International Symposium on Space Technology and Science, Nagoya, Japan, Paper No. 2013-e-18, June 2-9 (2013).

22. Abe, K.: An investigation of SGS-stress anisotropy modeling in complex turbulent flow fields, Flow, Turbulence and Combustion 92, 503-525 (2014).

23. Ohtsuka, T., Abe, K.: On the role of an anisotropy-resolving extra term for a subgridscale model in near-wall turbulence. Journal of Computational Science and Technology $\mathbf{7}$, 410-425 (2013).

24. Abe, K., Ohtsuka, T.: On the effect of an anisotropy-resolving subgrid-scale model on turbulent vortex motions. Proceedings of the 10th International Symposium on Engineering Turbulence Modelling and Measurements, Marbella, Spain, Paper No. S4-1, Sep. 17-19 (2014).

25. Bardina, J., Ferziger, J.H., Reynolds, W.C.: Improved subgrid scale models for large eddy simulation, AIAA Paper, No. 80-1357 (1980).

26. Inagaki, M.: A new wall-damping function for large eddy simulation employing Kolmogorov velocity scale, Int. J. Heat Fluid Flow 32, 26-40 (2011).

27. Abe, H., Kawamura, H., Matsuo, Y.: Surface heat-flux fluctuations in a turbulent channel flow up to $R e_{\tau}=1020$ with $\operatorname{Pr}=0.025$ and 0.71., Int. J. Heat Fluid Flow 25, 404-419 (2004)

28. Muto, M., Tsubokura, M., Oshima, N.: Negative Magnus lift on a rotating sphere at around the critical Reynolds number, Phys. Fluids 24, No. 014102 (2012).

29. Kim, J., Moin, P.: Application of a fractional-step method to incompressible NavierStokes equations. J. Comput. Phys. 59, 308-323 (1985).

30. Amsden, A.A., Harlow, F.H.: A simplified MAC technique for incompressible fluid flow calculations. J. Comput. Phys. 6, 322-325 (1970)

31. Rhie, C.M., Chow, W.L.: Numerical study of the turbulent flow past an airfoil with trailing edge separation. AIAA J. 21, 1525-1532 (1983).

32. Rajamani, B., Kim, J.: A hybrid filter approach to turbulence simulation, Flow, Turbulence and Combustion 85, 421-441 (2010).

33. Germano, M.: Properties of the hybrid RANS/LES filter, Theoretical and Computational Fluid Dynamics 17, 225-231 (2004).

34. Lee, M., Moser, R. D.: Direct numerical simulation of turbulent channel flow up to $R e_{\tau}$ $=5200$, J. Fluid Mechanics 774, 395-415 (2015).

35. Abe, K., Kondoh, T., Nagano, Y.: On Reynolds stress expressions and near-wall scaling parameters for predicting wall and homogeneous turbulent shear flows, Int. J. Heat Fluid Flow 18, 266-282 (1997). 
36. Abe, K., Kondoh, T., Nagano, Y.: A New Turbulence Model for Predicting Fluid Flow and Heat Transfer in Separating and Reattaching Flows - I. Flow Field Calculations, Int. J. Heat Mass Transfer 37, 139-151 (1994).

37. Abe, K., Jang, Y. J., Leschziner, M. A.: An investigation of wall-anisotropy expressions and length-scale equations for non-linear eddy-viscosity models, Int. J. Heat Fluid Flow 24, 181-198 (2003). 